\title{
Rock Particle Image Segmentation Based on Improved Normalized Cut
}

\author{
Gao Ting, Wang Weixing, Liu Wei and Yang Dandan \\ School of Information Eng., Chang'an University, China \\ Corresponding author: Wang Weixing, Email:wxwang@chd.edu.cn
}

\begin{abstract}
Rock particle images are different and complex, which is depending on various situations, in most cases, traditional image segmentation methods often make under-segmentation and over-segmentation. In order to overcome the disadvantages, a new algorithm based on improved graph based algorithm (Normalized Cut) is proposed. In the algorithm, to denoise and reduce data size, an image is reduced by scale transformation; and image segmentation is made by an improved Normalized Cut algorithm. In experiments, different types of rock particle images were tested, and the new algorithm was compared with the traditional algorithms such as Threshold, Edge detection, Minimum spanning tree, Clustering analysis, Fuzzy C-Means and Watershed etc. The testing results show that the image segmentation effect of the new algorithm is better than that by the traditional image segmentation algorithms. The studied algorithm was applied into the rock blasting in a large open-pit mine, and the results were satisfactory.
\end{abstract}

Keywords: rock particle; image segmentation; scale transformation; Normalized Cut

\section{Introduction}

Size and shape distributions of rock particles are the important factors for particle measurement in many rock engineering applications such as rock blasting, highway and railway construction, and building construction and so on, how to measure rock particles will directly affect the engineering quality and cost. The traditional manual method and mechanical sieving measurement method cannot provide the real-time analysis, but the machine vision system [1], which provides a kind of non-contact measurement, can do online analysis. In recent years, with the development of computer science, the image processing technology is gradually applied into the different research fields of the rock particle image processing [2-5]. Currently, Threshold method [6-7], Watershed [8-9], Edge detection [7], Clustering analysis[10], Fuzzy C-Means[11], Fuzzy clustering analysis methods ${ }^{[10-11]}$ and Graph based algorithms such as Minimum spanning tree [12-21] are often studied and used for rock particle image segmentation, but these methods are mainly based on gray level information, not on considering the combination of other information.

In this paper, an algorithm based on a graph algorithm (improved Normalized Cut) is used in the rock particle image segmentation, with the combination of image enhancement and multi-scale analysis. For some typical rock images, the new algorithm is compared with several widely used particle image segmentation algorithms, and is applied into the mining engineering, so as to verify the feasibility of the algorithm in the field of the rock particle image segmentation. 


\section{Basic Theory of Graph}

The clustering optimization [16] based on graph theory was studied in the 1960s firstly, and then, it was gradually applied in the field of image processing and analysis in the early 1980s. The image segmentation method based on graph theory is a top-down global segmentation method, which is robustness [12], and it combines the advantages of the graph method that is easy to process the local data characteristics and the advantage of objective function. The main idea of graph theory [12-14] is to map an image into a weighted undirected graph by taking each pixel as a node in graph, and to connect each pair of nodes by a graph edge. The weight on the graph edge corresponds to the neighboring relations between two pixels in the processed image. In the graph procedure, the image segmentation is completed by using various segmentation criteria. In the computing, a graph is regarded as a matrix, where each pixel corresponds to each element in the matrix. According to the value and position relationship of each element, a required classification can be got by using mathematical calculation. Figure 1 shows the mapping relation between an image and a graph.

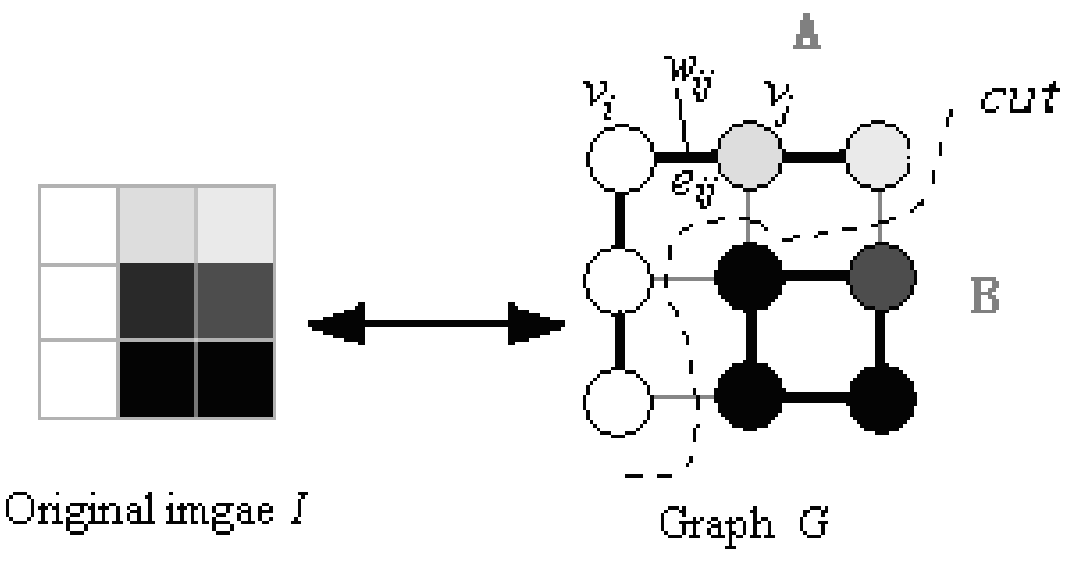

Figure 1. Mapping Relationship Between an Image and Its Graph

A graph $G=(V, E)$ can be partitioned into two disjoint sets, A and B, the two sets satisfy $A \cup B=V, A \cap B=\varnothing$, where $V$ is the vertex set and $E$ is the edge set. By simply removing edges connecting the two parts, the partition can be completed.

An optimal cut criterion is to maximize the internal similarity of two divided sub-graphs, to minimize the similarity of two sub-graphs, and to avoid skew segmentation regions in divided sub-graphs. Now the common cut criteria are as follows: Smart cut, Minimum Cut [14], Normalized Cut [15], Random Walk [16-17], Isoperimetric algorithm [17-18], Average cut [19-20] etc. In this study, we will mainly use the Minimum cut and Normalized cut.

\subsection{Minimum Cut}

A set of points in an arbitrary feature space can be represented as a weighted undirected graph ${ }^{G}=(V, E)$, ( $V$ for graph nodes and $E$ for graph sides). A node in the graph represents a point in the feature space; an edge is formed between every pair of nodes. The weight $w(i, j)$ on the graph edge connecting node $i$ and $j$ is the similarity between the two nodes. We can partition a graph $G=(V, E)$ into two mutually disjoint vertex sets $A$ and $B$ by removing the edges connecting the two parts. The degree of dissimilarity 
between these two sub-sets can be described as the total weight of the edges that have been moved. In graph theoretic language, it is called the cut:

$$
\operatorname{cut}(A, B)=\sum_{u \in A, v \in B} w(u, v)
$$

The goal for optimal bi-partitioning is to minimize this cut value; we name it for Minimum Cut [8]:

$$
\min \operatorname{cut}(A, B)=\sum_{i \in A, j \in B} w_{i j}
$$

The Minimum Cut seeks to partition a graph into k-sub-graphs, such that the maximum cut across the subgroups is minimized. This problem can be efficiently solved by recursively finding the minimum cuts that bisect the existing segments. The goal of the globally optimal criterion is to make total edge weights which connect the two minimum parts. According to the Min-Cut, there is a minimum similarity between two regions or classes:

However, as the minimum cut criteria tends to cut small sets of isolated nodes in graph, it cannot achieve the real best segmentation result. Figure 2 illustrates such a case, we see that the cut partitioning out node $\mathrm{n}_{1}$ or $n_{2}$ has a very small value on the right, which is smaller than the cut that partitions the nodes into the left and right halves. Min-cut 1 and Min-cut 2 are the isolated points by mistake:

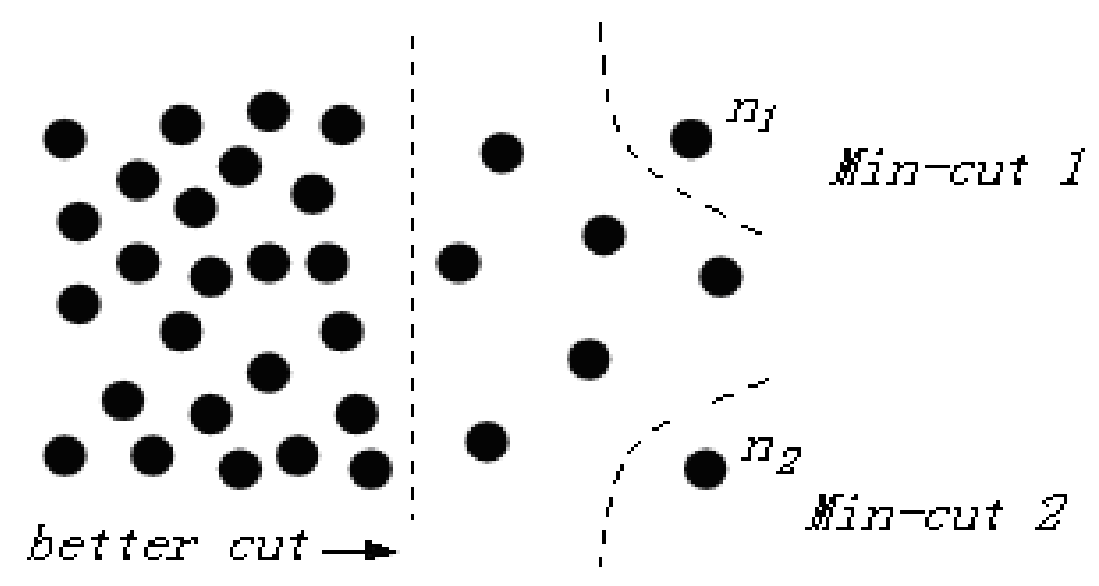

Figure 2. A Case where Minimum Cut Gives a Bad Partition

\subsection{Normalized Cut}

In order to avoid this unnatural bias for partitioning out small sets of points, Shi and Malik proposed a new method to measure the disassociation between two groups, which is based on Min-Cut. Instead of looking at the value of total edge weight connecting the two partitions, the method computes the cut cost as a fraction of the total edge connecting to all the nodes in graph. We call it Normalized Cut (NCut) [21-21]:

$$
N C u t(A, B)=\frac{\operatorname{cut}(A, B)}{\operatorname{assoc}(A, V)}+\frac{\operatorname{cut}(A, B)}{\operatorname{assoc}(B, V)}
$$

Where, ${ }^{a \operatorname{ssoc}(A, V)}$ is the total connection from nodes in $A$ to all nodes in graph, and $\operatorname{assoc}(B, V)$ is similarly defined: 


$$
\begin{aligned}
& \operatorname{assoc}(A, V)=\sum_{u \in A, t \in V} w(u, t) \\
& \operatorname{assoc}(B, V)=\sum_{u \in B, t \in V} w(u, t)
\end{aligned}
$$

With this definition of the disassociation between the groups, the cut that partitions out small isolated points will no longer have small NCut value, since the cut value will almost be a large percentage of the total connection from that small set to all other nodes. We see that the cut 1 value across node ${ }^{\mathrm{n}_{1}}$ will be $100 \%$ of the total connection from that node, the same as cut2, so Normalized Cut can avoid small sets of points.

The Minimizing Normalized Cut is eventually to be NP-complete. In order to reduce the NP problem, Shi and Malik changed the problem of computing the optimal NCut value to the problem of solving the eigenvalue and eigenvector of a matrix, using the spectral graph theory to solve the cost function approximately.

Let $D$ be an $N^{*} N$ diagonal matrix with d on its diagonal, $W$ be an $N^{*} N$ symmetrical matrix with ${ }_{i j}=w_{i, j}, \quad \mathrm{k}=\frac{\sum_{x_{i}>0} \boldsymbol{d}_{i}}{\sum_{i} \boldsymbol{d}_{i}}$ and $I$ be an $N^{*} I$ vector. The diagonal elements of $D$ are as:

$$
d_{i j}=\sum_{j} w_{i j}
$$

Then, setting $\boldsymbol{y}=(\mathrm{I}+\boldsymbol{x})-b(\mathrm{I}-\boldsymbol{x})$, the NCut formula can be presented as:

$$
\operatorname{NCut}(A, B)=\operatorname{NCut}(\boldsymbol{x})=\frac{\boldsymbol{y}^{T}(\boldsymbol{D}-\boldsymbol{W}) \boldsymbol{y}}{\boldsymbol{y}^{T} \boldsymbol{D} \boldsymbol{y}}
$$

We can change solving the problem of minimum NCut value into solving the problem of finding a $\boldsymbol{y}$ value that can minimize characteristic equation. If $\boldsymbol{y}$ is relaxed to take on real values, we can simplify the last type to:

$$
(\boldsymbol{D}-\boldsymbol{W}) \boldsymbol{y}=\lambda \boldsymbol{D} \boldsymbol{y}
$$

The Normalized Cut method uses the second smallest eigenvector of the generalized eigensystem (8) as the real valued solution to solve the Normalized Cut problem. Confirming a segmentation value in vector $y$ makes the node greater than this value belongs to $A$, the rest belong to $B$, and the corresponding image segmentation is accomplished. However, as the nature of normalized cut is to cluster the points of image data, with the increase of image size, the computation of the method will be sharply increased and the segmentation speed will be slower. Hence the Normalized Cut is rarely simply used to partition images.

\section{Segmentation on Multi-Scale and Improved Normalized Cut}

\subsection{Algorithm Overview}

In general, for the segmentation of rock particle images [15], there are many difficulties such as uneven size, overlapping, complex texture and low light contrast between background and objects. If we solely process images based on gray levels, parts of the background and objects will be mixed as a fault object. The image segmentation method based on graph theory focuses on the global and local data processing, which considers not only the pixel gray level relationship, but also the spatial relationship between pixels, and the method can get a satisfactory result.

The image segmentation method based on graph theory just considers the similarity between pixels in an image, not the internal textures of an object. As the nature of this 
method is to cluster image data, the larger image size is, the more computing time is needed, and the slower segmentation speed is. In order to decrease the computing time of Normalized Cut (an algorithm on graph), we improve the algorithm from two aspects: starting from pixels, on the premise of little effect on the segmentation performance, and the scale transformation is carried out appropriately. Choosing appropriate pixels from an original image makes the scaled-down image keep the general characteristics of the original image as far as possible, and reduces the computing time through the coarse image. Meanwhile, starting from the Normalized Cut algorithm itself, an appropriate weighting function is chosen to make the similarity matrix sparse [18], then, the Lanczos method is used to solve the characteristic vector. Besides, the traditional Normalized Cut acts on gradient magnitude, not gray level.

\subsection{Image Pre-processing}

Generally, in Mining engineering, the rock particle image is complex and the image quality is lower, under different illumination conditions, where serious noise and fuzzy edges exist in the images. So, before image segmentation, the image enhancement process is needed first. While the traditional linear filter can filter away some noise, it can also filter out parts of marginal information on low frequency and cause edge blur, such as Wiener filtering and Gaussian filtering. As one kind of nonlinear filter, the Bilateral filter [23-25] is proposed on the basis of the Gaussian filter. While considering the space relation of pixels, it also considers the relationship between gray levels. The Bilateral filter achieves the goal of keeping edge information and filtering away some noise simultaneously.

The Bilateral filter was firstly proposed by Tomasi, and it was composed of two functions, one function's filter coefficient is determined by a geometrical distance, while another is determined by the difference of pixel levels. The Bilateral filter has a Gaussian

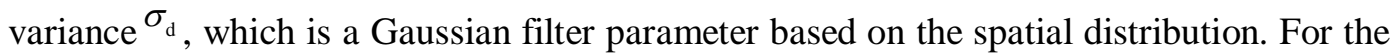
edges, the further distance between pixels and edge is, the smaller influence on edge pixels is. It can guarantee the preservation of pixels near the edges.

The theoretical formula is as:

$$
\mathrm{h}(\mathrm{X})=k^{-1}(\mathrm{X}) \int_{-\infty}^{\infty} \int_{-\infty}^{\infty} f(\xi) c(\xi, \mathrm{X}) s(f(\xi), f(\xi)) d \xi
$$

The discrete formula can be written as:

$$
\mathrm{h}(\mathrm{X})=k^{-1}(\mathrm{X}) \sum_{\Omega} f(\xi) c(\xi, x) s(f(\xi), f(\xi))
$$

The Gaussian weight $c(\xi, \mathrm{X})$ based on the spatial distance and the Gaussian weight $s(\xi, \mathrm{X})$ based on the similarity degree between pixels can be expressed respectively as follows:

$$
\begin{aligned}
& c(\xi, \mathrm{X})=e^{-\frac{1}{2}\left(\frac{d(\xi, \mathrm{X})}{\sigma_{d}}\right)^{2}} \\
& s(\xi, \mathrm{X})=e^{-\frac{1}{2}\left(\frac{\sigma(f(\xi), f(X))}{\sigma_{r}}\right)^{2}}
\end{aligned}
$$

Where

$$
\begin{array}{r}
d(\xi, \mathrm{X})=d(\xi-\mathrm{X})=\|\xi-\mathrm{X}\| \\
\sigma(\phi, f)=\sigma(\phi-f)=\|\phi-f\|
\end{array}
$$


$d(\xi, \mathrm{X})$ is the Euclidean distance and $\sigma(\phi, f)$ is the gray level difference between two pixels. $\sigma_{d}$ and $\sigma_{r}$ are respectively the spatial proximity factor and the proximity factor of brightness, they are both based on the standard deviation of the Gaussian function, and have a strong effect on filtering, so before filtering, these values are confirmed according to the rock particle size.

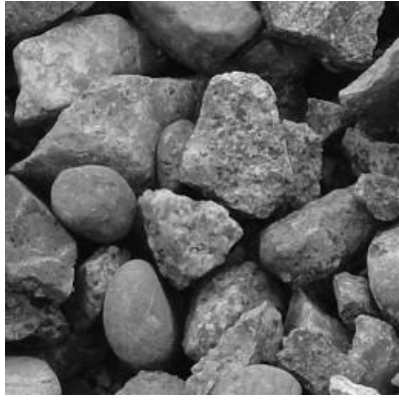

(a)

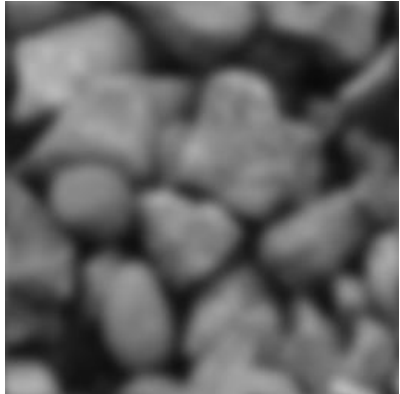

(b)

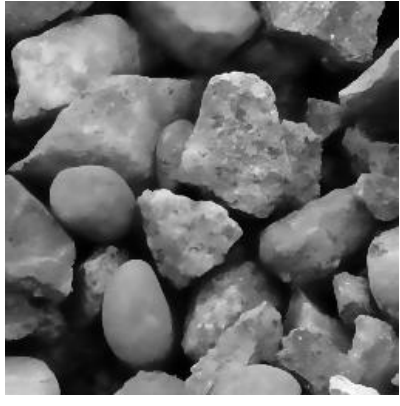

(c)

Figure 3. Comparison of Rock Particle Image Preprocessing; (a) Original Image Taken on a Muckpile; (b) Result of Gaussian Filtering, its Template Radius is 10; and (c) Result of Bilateral Filtering, its Template Radius is 10,

$$
\sigma_{d}=20 \quad \sigma_{r}=0.1
$$

Figure. 3 shows rock particle image filtering using the Gaussian filter and the Bilateral filter. Two filters are set to the same values: the template radius is 10, the proximity factor of brightness is $\sigma_{r}=0.1$. From the comparison in Figure3, we can see that in image(c), the noise on the rock surface is filtering out and the edge information is kept well while image (b) has been blurred. The Bilateral filtering is better than the Gaussian filtering.

\subsection{Multi -scale Transformation}

Since the size of many collected rock particle images is too big to handle, in order to make the processing more rapid, the image size should be scaled down. The multi-scale analysis can synthesize pictorial information at different scales; unify the accuracy at fine scale and the easy segmentation at coarse scale. With the processing at small scale, the number of pixels follow-up to participate in the operation can be reduced.

The Bicubic interpolation [24] (cubic convolution method) can overcome the problems of the nearest neighbor interpolation in which the image quality is low and the object contours are fuzzy. The Bicubic interpolation is a complex interpolation method, it uses 16 points' gray values to process the cubic interpolation, and these points are around the sampling site. The method considers not only the gray level effect of 4 direct adjacent points, but also the effect of gray level variance rate between neighboring points. Considering one floating point coordinate, we select $S(\omega)$ as the primary function, the bicubic interpolation formula is:

$$
f(i+u, j+v)=\boldsymbol{A B C}
$$

Where, matrix $A, B, C$ are respectively as follows:

$$
\boldsymbol{A}=\left[\begin{array}{llll}
S(1+u) & S(u) & S(1-u) & S(2-u)
\end{array}\right]
$$




$$
\begin{gathered}
\boldsymbol{B}=\left[\begin{array}{cccc}
f(i-1, j-1) & f(i-1, j) & f(i-1, j+1) & f(i-1, j+2) \\
f(i, j-1) & f(i, j) & f(i, j+1) & f(i, j+2) \\
f(i+1, j-1) & f(i+1, j) & f(i+1, j+1) & f(i+1, j+2) \\
f(i+2, j-1) & f(i+2, j) & f(i+2, j+1) & f(i+2, j+2)
\end{array}\right] \\
\boldsymbol{C}=\left[\begin{array}{c}
S(1+v) \\
S(v) \\
S(1-v) \\
S(2-v)
\end{array}\right]
\end{gathered}
$$

The primary function is

$$
S(\omega)=\frac{\sin (\omega)}{\omega} \approx\left\{\begin{array}{c}
1-2|\omega|^{2}+|\omega|^{3},|\omega|<1 \\
4-8|\omega|+5|\omega|^{2}-|\omega|^{3}, 1 \leq|\omega|<2 \\
0,|\omega| \geq 2
\end{array}\right.
$$

The Bicubic interpolation can be close to a high definition picture; the scale-down picture not only has fewer pixels, but also makes a reservation of high frequency components. The following pictures show the comparing effect between the original image and the bicubic interpolation image shrinking one time:
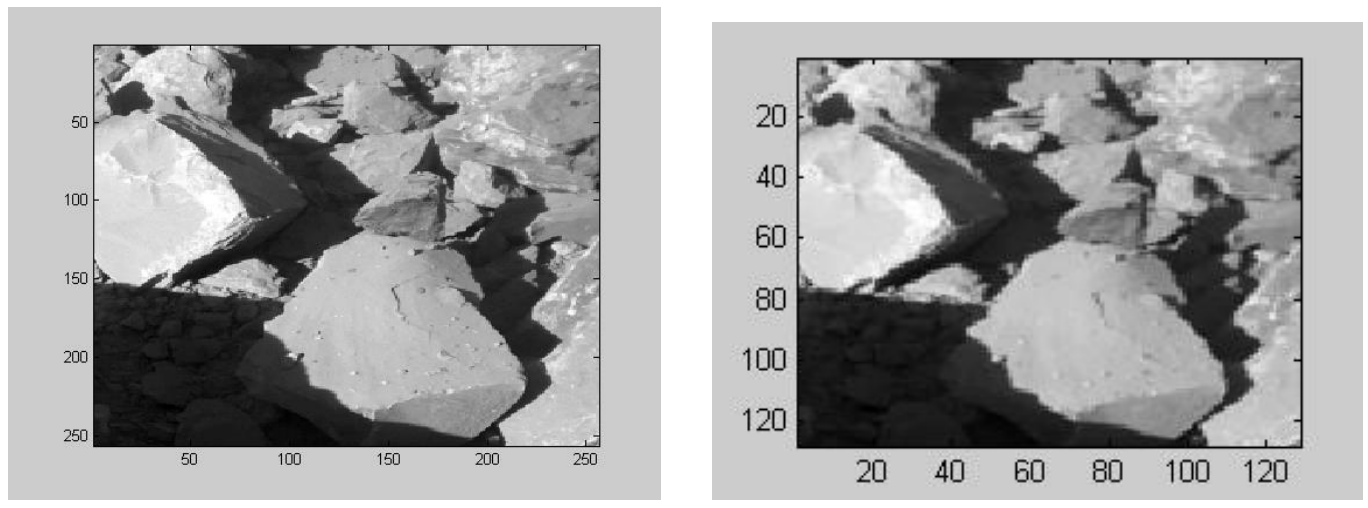

Figure 4. Two Different Images: Original Image (Left); Processing Image Shrinking One Time (Right)

In Figure4, the height and width of the original image is shrunk one time by the Bicubic interpolation method. We stretch the processing image on the right to the same size as the original image, and it is easy to find that the method keeps a lot of useful edge pixels while reducing the number of pixels. We just need to process what we want in the scale-down image.

\subsection{Improved Normalized Cut Image Segmentation Algorithm}

After the scale-down transformation, the size of image and the number of pixels are both changed. We use the improved Normalized Cut in the scale-down image for image segmentation. The traditional Normalized Cut directly acts on the nodes in graph, the relationship between the gray value of nodes and space distance are the main parameter of image segmentation. Commonly, a rock particle image has a sharp edge, where the gradient magnitude value is obvious and larger, to detect the target edges preferably, the 
traditional Normalized Cut which acts on the gray values of pixels will be changed to act on the gradient magnitude values of pixels. The specific steps are as follows:

(1) Work out the second derivative through a parity filter; build a gradient magnitude image;

(2) For the gradient magnitude image, set up a weighted graph ${ }^{G}=(V, E)$, and set the weight on the edge connecting two nodes being a measure of the similarity between the two nodes. The weight $w_{\mathrm{i}, j}$ is defined on the basis of the gradient magnitude $F$ and ubiety $X$ :

$$
w_{i, j}=e^{\frac{-\left\|\boldsymbol{F}_{(i)}-\boldsymbol{F}_{(j)}\right\|_{2}^{2}}{\sigma_{I}}} *\left\{\begin{array}{cc}
e^{\frac{-\left\|\boldsymbol{X}_{(i)}-\boldsymbol{X}_{(j)}\right\|_{2}^{2}}{\sigma_{X}}} & \text { if }(D(i, j)<r) \\
0 & \text { else }
\end{array}\right.
$$

Where, $D(i, j)$ is the Euclidean distance [25] between the two nodes:

$$
D(i, j)=\left\|X_{(i)}-\mathrm{X}_{(\mathrm{j})}\right\|_{2}=\sqrt{\left(x_{i}-x_{j}\right)^{2}+\left(y_{i}-y_{j}\right)^{2}}
$$

Where, $r,{ }^{\sigma_{I}}, \sigma_{x}$ are the man-made setting parameters, through introducing the parameter $r$, we can directly judge the two nodes whose distance $D(i, j)$ is larger than $r$, and just compute $w_{\mathrm{i}, j}$ between two nodes, their distance is less than $r$. For different images, $\sigma_{I}$ or $\sigma_{x}$ may cause different image segmentation effects, the values of them cannot be too large or too small, the product of them should be $10 \%-20 \%$ of the feature distance function in the total range.

(3) Build a similarity matrix $\bar{W}$ and make it sparse

(4) Solve the formula (8), for eigenvectors with the smallest eigenvalues. The eigenvector with the second smallest eigenvalue is the continuous approximation to the discrete bi-partitioning indicator vector that we seek.

(5) Partition graph using the eigenvectors completely.

\subsection{Procedure of Rock Particle Image Segmentation Algorithm}

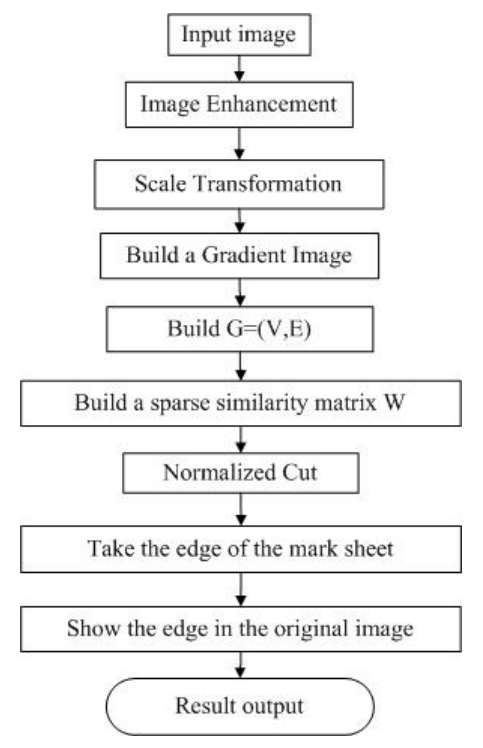

Figure 5. Algorithm Flow Chart 
After calculating related parameters, the Normalized Cut determined by the eigenvector and eigenvalue partitions an image to multiple parts, we name which as a mark sheet; makes the border detection for the mark sheet, extracts edges and fuse it with the original interpolation image. The whole algorithm flow chart is in the above.

\section{Experiments}

According to the number of rock particles in an image and different applications, three types of rock particle images are tested. They are: Crashed particles (aggregates) from a falling stream; Blasted rock particles (fragments) from muckpiles; and natural rock particles (aggregates). The algorithm proposed in the paper is used in these particle images, and it is compared with some widely used methods, such as Auto-threshold segmentation [3, 6-7], Contour extraction based on priori knowledge [26-30], Minimum spanning tree segmentation [21], FCM segmentation [11-12], Clustering segmentation [10] and Watershed segmentation [8-9]. The testing results show that the improved Normalized Cut is suitable both for densely packed rock particle images and sparsely distributed particle images, and the segmentation results are satisfactory.

\subsection{Test for Sparsely Distributed Rock Particle Images}

When crashed rock particles are transported from a conveyor belt to a pile, one simple way to take images online is to take images from a gravitational falling stream, and the image is acquired by using back lightning, in which way, particles are sparsely distributed on an image. The rock particle images have a strong differentiation between the target and background.

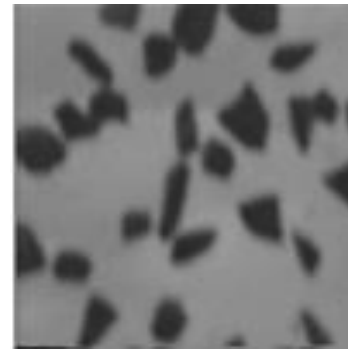

(a)

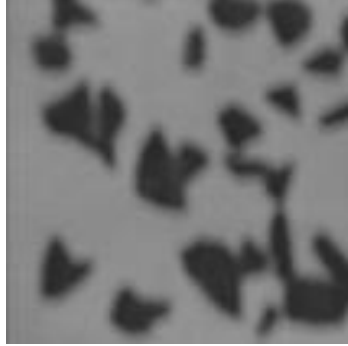

(e)

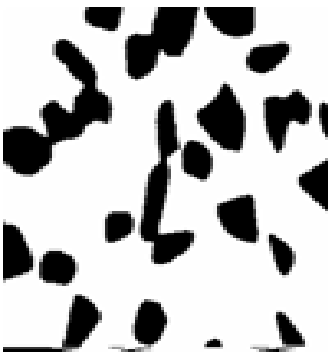

(b)

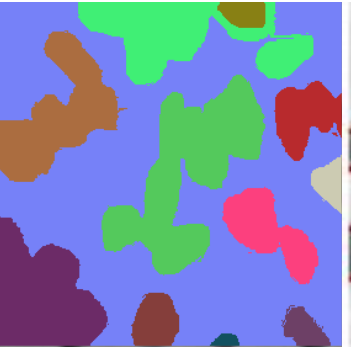

(c)

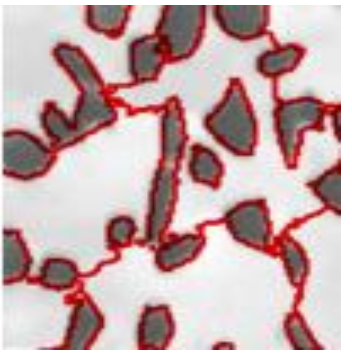

(d)

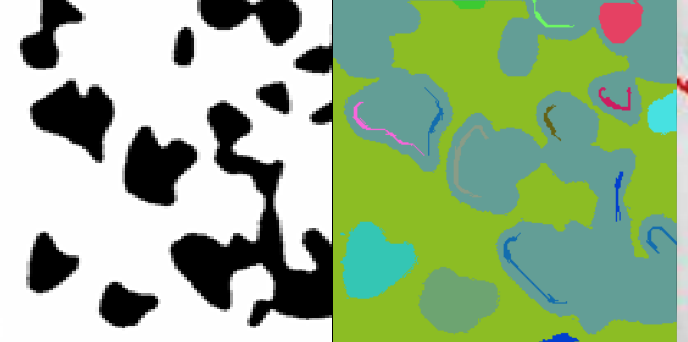

(f) (g)

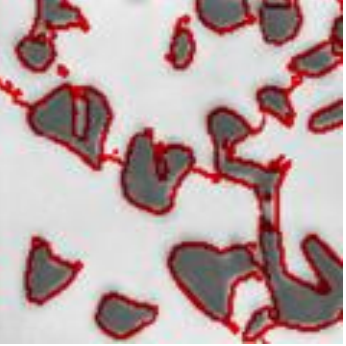

(h)

Figure 6. Various Image Segmentation Results on Falling Stream Rock Particle Images \#1 (a), (e) Original Images; (b), (f) OTSU Thresholding Results; (c), (g) MST Results; and (d), (h) Improved Graph-Segmentation Results

Due to the back lightning, the surface information of particles is missing, the contours of most particles are clear, which is good for image binarization. In some cases, the image might be blurred in some parts or particles touching each other because of unstable 
particle moving speed. However, the particle adhesion phenomenon (particles touching form clusters) exists in some parts.

In Figure6(a), there are 25 particles of different sizes and shapes, and the grey scales are different between particles and background. An auto- thresholding algorithm can well separate the particles from background for most parts, but not for the adhesion parts, as shown in the image in Figure6(b), there are at least three clusters including 2, 3 and 4 particles respectively. Minimum spanning tree segmentation algorithm separates the targets from background, but the targets include 6 clusters, the clusters include some background parts, therefore the clusters are larger, as shown in the image in Figure6(c). The algorithm in this study can split some rock particle clusters with certain level, only 2 clusters are in the image.

The image in Figure6(e) has 25 particles of different sizes and shapes, and the grey scales are different between particles and background. An auto- thresholding algorithm can well separate the particles from background for most parts, but not for the adhesion parts, as shown in the image in Figure6(f), there is a large cluster which is formed by 9 particles, in addition, there are other four clusters, each of these including 2 particles. Minimum spanning tree segmentation algorithm separates the targets from background, but the image include 3 large clusters that include 4, 4 and 11 particles respectively, as shown in the image in Figure6(g). The algorithm in this study can split some rock particle clusters with certain level, and only 3 clusters, including 2, 2 and 6 particles, are in the image.

In conclusion, for a sparsely packed particle image with back lightning, if the grey levels in adhesion parts are different enough from that in the touching particles, the improved graph based algorithm can separate the touching particles well, and it is superior to the former grey level similarity algorithms, and much better than traditional graph based algorithm. For some grey levels in adhesion parts being slightly different to the touching particles, the segmentation result should be improved more by using binary image segmentation algorithms.

\subsection{Test for Densely Packed and Similar Sized Rock Particle Images}

In aggregate production and mining industry, rock is blasted and crashed into rock particles of different sizes and shapes, then natural rock particles are mixed with crushed aggregates, therefore, the particle distribution is measured and analyzed on a conveyor belt or a muckpile or a gravitational falling stream as presented above, to evaluate the particle quality for different applications. Figure 7(a) presents a typical natural rock particle image in a muckpiles, and the image in Figure8(a) includes both natural particles and crushed particles in a muckpile. The two images are different to the images in Figure6(a, e). In each of the images, a natural particle has a smooth surface, and a crushed particle has a rough surface. The particles in an image are densely packed, touching and overlapping each other. In any of the images, different colors and 3D geometry make particles have different grey scales; and some void spaces are among the particles. All the properties make image segmentation difficult, we take the following two images for different algorithm comparison to validate the new algorithm.

Rock particles in Figure7 and Figure8 are from muckpiles, where the particle touching problem exists. Otsu operated in images (b) can roughly partition the image into particles, the void spaces are separated into background, some small particles are also become background, particles touching or overlapping each other, anyhow, it may easily cause under-segmentation; Although Contour based algorithm operated in images (c) can give a fair image segmentation result, but it causes the discontinuous edges, create both over-segmentation and under-segmentation problems; Minimum spanning tree segmentation algorithm is good for blurring images, but not for such particle images, the extracted particles are larger than what they are, therefore, the under-segmentation problem happen in (d); FCM result (e) is similar to that in (b), but more detailed 
information reflects on the particle surfaces, with less under-segmentation problems; and Clustering analysis based algorithm is also not suitable for this kind of rock particle images, the segmentation effect is better than that in (d), just having some under-segmentation problems in the regions of small particles; Watershed algorithm operated in images is similar to that in (c), some problems of under-segmentation and over-segmentation still exist. The algorithm in this paper can solve the over-segmentation problem, the main particles are extracted, and the under-segmentation is less by comparing with the other algorithms.

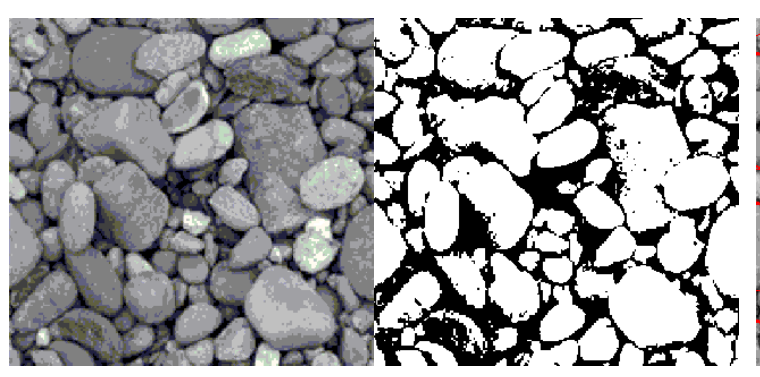

(a) (b)

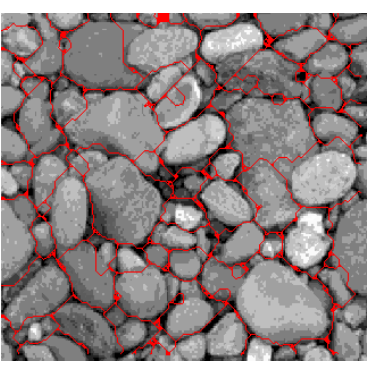

(c)

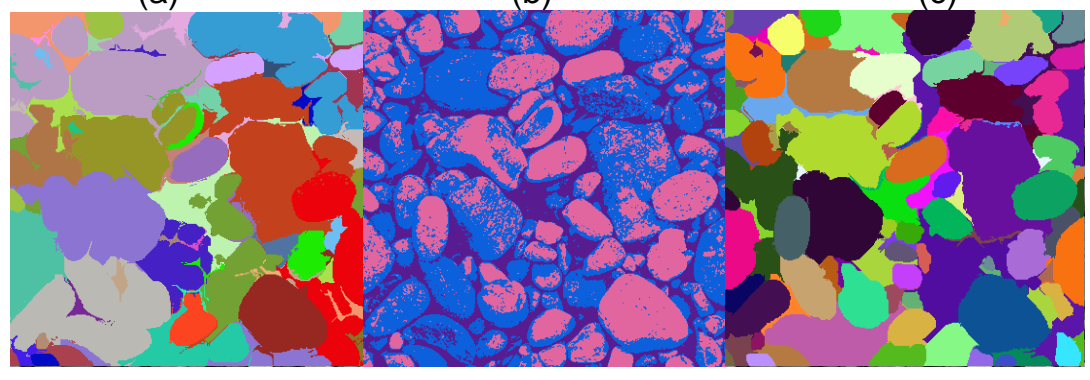

(d)

(e)

(f)

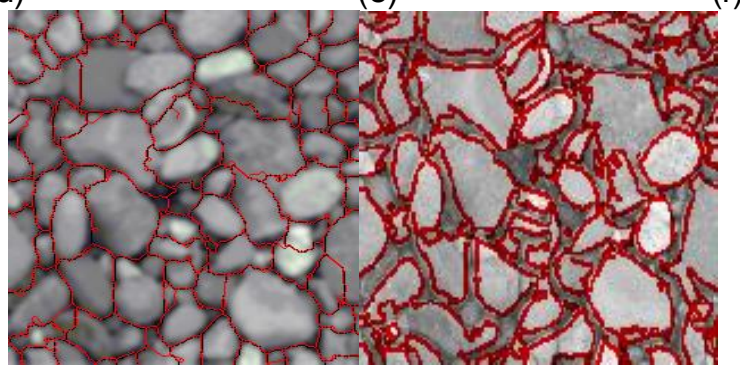

(g)

(h)

Figure 7. Different Algorithm Results for Natural Rock Particle Image \#1: (a) Original Image; (b) Auto-Thresholding Result; (c) Contour Extraction Result; (d) MST Segmentation Result; (e) FCM Result; (f) Clustering Analysis Result; (g) Watershed Segmentation Result; and (h) Improved Graph Segmentation Result

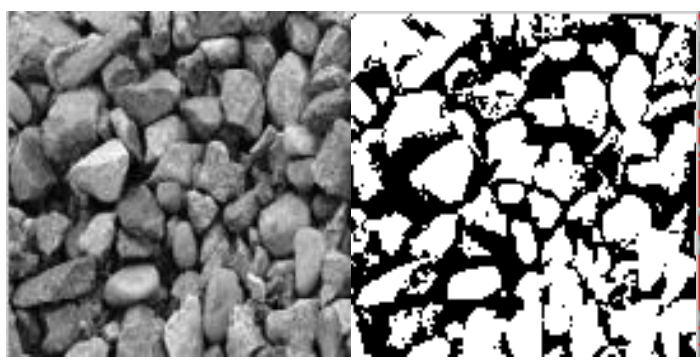

(a) (b)

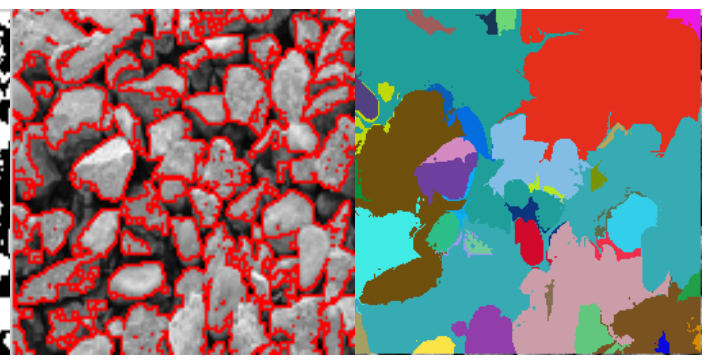

(c) (d) 


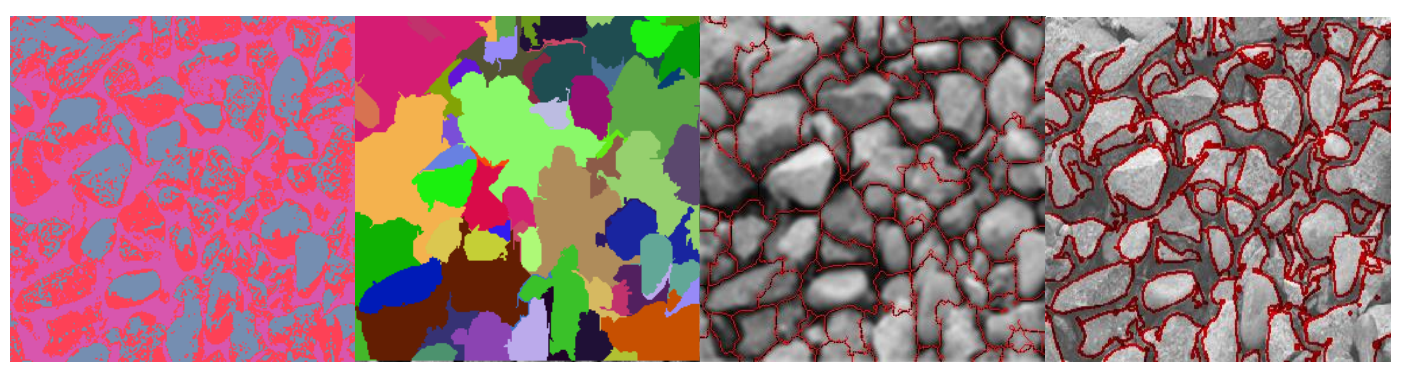

(e)

(f)

(g)

(h)

Figure 8. Different Algorithm Results for Natural Rock Particle Image \#2: (a) Original Image; (b) Auto-Thresholding Result; (c) Contour Extraction Result; (d) MST Segmentation Result; (e) FCM Result; (f) Clustering Analysis Result; (g) Watershed Segmentation Result; and (h) Improved Graph Based Image Segmentation Result

However, the natural particle images are easier processed than non-natural particle images, no matter what kind of particle images, the new algorithm is better than the others. To show the detailed information of the image segmentation for crushed aggregates or fragmental images, Figure9 gives 4 examples more.

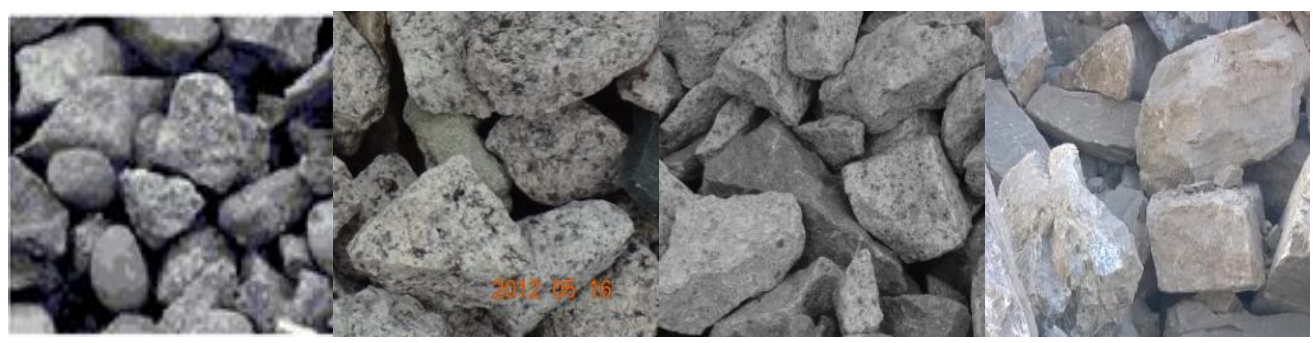

(a)

(b)

(c)

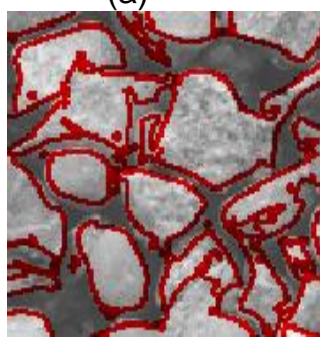

(e)

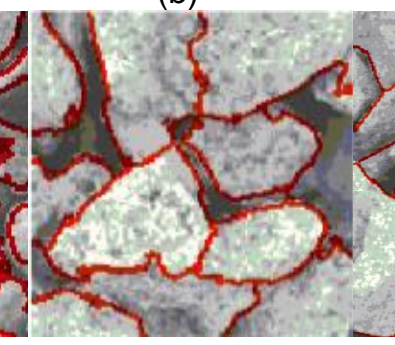

(f)

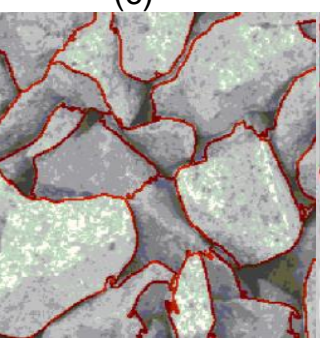

(g) (d)

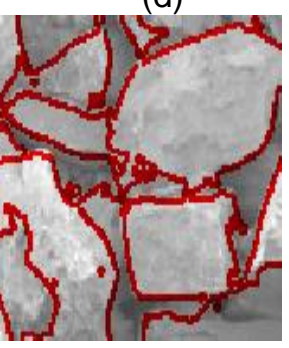

(h)

Figure 8. Image Segmentation Results of 4 Muckpile Rock Particle Images by Using Improved Graph Based Image Segmentation Algorithm: the First Row (a, b, c, d) is for 4 Original Images, and the Second Row $(e, f, g, h)$ is for the Corresponding Image Segmentation Results

The rock particles in Figure9 are from 4 different muckpiles, and they have various shapes and rich textures on surfaces. There are some void spaces among particles in the images. For this type of rock particle images, the main problem is whether we can handle various adhesion parts. Since some rock particles are of dark colors, a threshold segmentation algorithm is easy to make some particles into background, therefore, it is unable to handle the adhesion problem; the algorithm for contour extraction still cannot handle the particle adhesion parts, and may create some false edges as the contours of particles; the results of Minimum spanning tree segmentation algorithm are bad in this cases; FCM segmentation algorithm and Clustering analysis algorithm are also not 
suitable for this kind of rock particle images as analyzed above; Watershed image segmentation algorithm can make good segmentation results, but the problems of under-segmentation and over-segmentation still exist and the contour positions are inaccurate; and the segmentation algorithm proposed in this study is superior to the formers, despite some noise interference, it can split adhesion parts as presented in Figure9.

\subsection{In Conclusion}

The images of the parsley packed particles are easy to segment by a traditional algorithm if without particle touching problem, therefore the new algorithm is good for resolving touching problem caused by light difference between particles.

For the particle images of rough surfaces, particles are densely packed, such as crushed particles or fragments, the new algorithm is better than the others too. In the muckpile surface, rock particles (fragments) are touching or overlapping each other, which make image segmentation hard. Compared with the natural rock particle images, the rock fragment edges are more complicated and the sizes of fragments vary much. As the existence of noise and shadows, Otsu segmentation effects are not good; in contrast, the contour extraction in images can extract the edges of fragments, but the algorithm is unable to handle the adhesion problem; the segmentation effects of Minimum spanning tree are too bad; the segmentation effects of FCM are the similar to that by Otsu and Watershed algorithms, and it is unable to handle the adhesion problem; Clustering analysis based algorithm is not suitable for this kind of images; Watershed algorithm can roughly partition the image into particles, but the under-segmentation and over-segmentation problems still exist; and the algorithm in this paper can handle the adhesion problem, even though there exist some over-segmentation problem in some parts in an image, the segmentation effects are superior to that by Watershed algorithm.

The experimental results show that in the rock particle image segmentation, the studied algorithm based on graph theory and multiscale transform is superior to the traditional particle image segmentation algorithms for both sparsely and densely packed particle images, and for both natural particle and crushed (fragmented) particle images. The proposed algorithm can roughly partition the targets, and subdivide the adhesion parts. Though the segmentation effect is not $100 \%$ well, it can meet a variety of rock particle image segmentation requirements in real applications.

\section{Conclusions}

The study of the image segmentation algorithm based on graph theory is to map an image to a weighted undirected graph, and to convert the image segmentation problem to the graph segmentation problem. Normalized Cut (NCut) can synthesize information such as color, texture and distance, and can synthesize the local characteristics while grasping the global information of an image. In this paper, the image is set to multi-scale transform first, then the improved Normalized Cut method is used in the rock particle image segmentation, and finally the segmentation result is concluded by the image fusion of the original image and NCut segmentation image. By comparing among different rock particle images, we get the result that what kinds of rock particle images are more suitable for NCut algorithm, as above discussed. The segmentation algorithm based on graph theory has a certain advantage comparing to the traditional algorithms in the rock particle image segmentation, which can decrease the effect of the textures on the rock particle surface. The algorithm of NCut can make a good segmentation effect on a few kinds of rock particle images which are used in experiments. Changing relevant parameters can improve the image segmentation effect, but how to decrease the computing time is still a problem we need to solve in the near future. For the special particle images, the information fusion technology may be used [10]. 


\section{Acknowledgements}

This research is financially supported by the National Natural Science Fund in China (grant no. 61170147), the Special Fund for Basic Scientific Research of Central Colleges (No. 2013G2241019), and the Shaanxi Province Science \& Technology Fund (No. 2013KW03).

\section{References}

[1] Cassidy R, Morrow P J, McCloskey J. A machine vision system for quantifying velocity fields in complex rock models[J].Machine Vision and Applications, (2006), 16(06):343-355.

[2] Wang Weixing, (2006), Image analysis of particles by modified Ferret method - best-fit rectangle, International Journal: Powder Technology, Vol. 165, Issue 1, pp. 1-10.

[3] Wang, W.X., (1999), Image analysis of aggregates, International Journal: Computers \& Geosciences 25, 71-81.

[4] Wang, W.X., (1998), Binary image segmentation of aggregates based on polygonal approximation and classification of concavities. International Journal: Pattern Recognition, 31(10), 1503-1524.

[5] W X Wang, W S Li and X Yu, (2012), Fractional differential algorithms for rock fracture images, The Imaging Science Journal,Vol.60, IMAG 93 @ RPS 2012, pp.103-111.

[6] Gruzman I S. Threshold binarization of images based on the skewness and kurtosis of truncated distributions[J]. Optoelectronics, Instrumentation and Data Processing, (2013), 49(03):215-220.

[7] Weixing Wang, (2011), Colony image acquisition system and segmentation algorithms, Optical Engineering, 50(12), 123001 (Nov 29, 2011); doi:10.1117/1.3662398.

[8] Makrogiannis S, Vanhamel Iris, Fotopoutos S, Watershed-based multiscale segmentation method for color images using automated scale selection[J]. Journal of Electronic Imaging, (2005), 14(03): 1-16.

[9] Wang Weixing and Chen Liangqin, (2015), Flotation bubble tracing based on Harris corner detection and local gray value minima, Minerals, (2015), 5(2), 142-163, doi: 10.3390/min5020142.

[10] Hathaway R J, Yingkang Hu. Density-Weighted Fuzzy C- Means Clustering[J]. Fuzzy Systems, IEEE Transactions on, (2009), 17(01): 243-252

[11] Wang Lingfeng, Pan Chunhong. Robust level set image segmentation via a local correntropy-based K-means clustering[J]. Pattern Recognition, (2014), 47(05): 1917-1925.

[12] Qian Yuntao, Zhao Rongchun, pretty letter. Robust clustering - the method based on graph theory and objective function [J]. Journal of electronics, 1998, 2:91-94

[13] BAJGER M, MA F. Efficient graph-based image segmentation[J]. Computer Vision, (2004), 59(2): 167-181

[14] Z Wu, R Leahy. An optimal graph theoretic approach to data clustering: theory and its application to image segmentation[J]. IEEE Trans PAMI, (1993), 15(11): 1101-1113

[15] Gedeon Tomas, Parker Albert E, Campion Collette, Annealing and the normalized-cut[J]. Pattern Recognition,(2008), 41(02): 592-606

[16] Grady L. Random walks for image segmentation [J]. Transactions on Pattern Analysis and Machine Intelligence, (2006), 28(11): 1768-1783

[17] Grady L, Schwartz E L. Isoperimetric partitioning: A new Algorithm for graph partitioning[J]. SIAM Journal on Scientific Computing, (2006),27(06): 1844-1866

[18] L J Grady. Space-Variant Computer Vision: A Graph-Theoretic Approach[D]. Boston Univ,(2004)

[19] S Sarkar, K L Boyer. Quantitative Measures of Change Based on Feature Organization: Eigenvalues and Eigenvectors[C]. Computer Vision and Pattern Recognition, (1996)

[20] J Shi, J Malik. Normalized Cuts and image segmentation[J]. IEEE Trans PAMI, (2000), 22(08): 888-905.

[21] Wang Weixing, Zhang Xin, Cao Ting, Tian Liping, Liu Sheng, Wang Zhiwei, (2014), Fuzzy and Touching Cell Extraction on Modified Graph MST and Skeleton Distance Mapping Histogram, Journal of Medical Imaging and Health Informatics, Vol. 4, No. 3, 2014, 2156-7018/2014/4/001/008, doi:10.1166/jmihi.(2014). 1264.

[22] Andra H,Combaret N, Dvorkin J, Digital rock physics benchmarks-Part I: Imaging and segmentation[J]. Computers and Geosciences, (2013), (50): 25-32

[23] C Tomasi, R Manduchi. Bilateral filtering for gray and color images[A]. Proceedings of the 6th International Conference on Computer Vision [C]. New Delhi: IEEE Computer Society,(1998): 839-846

[24] Gao Shengkui, Gruev V. Bilinear and bicubic interpolation methods for division of focal plane polarimeters[J]. Optics Express, (2011),19(27): 26161-26173

[25] Chen Ling. Optimal algorithm for complete Euclidean distance transform[J]. Chinese Journal of Computers, (1995), 18(08): 611-614

[26] PF Felzenszwalb, DP Huttenlocher. Efficient graph-based image Segmentation[J]. International Journal of Computer Vision, (2004), 59(02): 167-181. 
[27] Wang Weixing, (2008), Fragment Size Estimation without Image Segmentation, International Journal: Imaging Science Journal, Vol. 56, p.91-96, April, 2008.

[28] W.X. Wang, F. Bergholm, B. Yang, (2003), Froth delineation based on image classification, International Journal of Mineral Engineering, Volume 16, Issue 11 , November (2003), Pages 1183-1192.

[29] LI Xu, XU Shu-chang, YOU Yu-cai, Segmentation method for personalized American car plate based on clustering analysis [J]. Journal of Zhejiang University (Engineering Science), (2012), 46(12): 2155-2159.

[30] Wang Weixing, Wang Fengping, Huang Xiaojun, Song Junfang. Rock fracture image acquisition using two kinds of lighting and fusion on a wavelet transform, Bulletin of Engineering Geology and the Environment, Online ISSN 1435-9537, DOI 10.1007/s10064-015-0747-4, (2015), 05, Publisher: Springer Berlin Heidelberg.(EI: 20151800813021). 
International Journal of Control and Automation Vol.10, No.4 (2017) 